\title{
On the occasion of Manfred W. J. Gerlach's 60th anniversary
}

\author{
Andreas Warnke · Peter Riederer - Marcel Romanos
}

Published online: 1 August 2014

(C) Springer-Verlag Wien 2014

Manfred Gerlach is the founding editor in chief of this journal. This issue is dedicated to him as an outstanding personality in the fields of developmental child and adolescent as well as adult psychiatry and neurology. His scientific opus represents an extremely broad and unique scientific spectrum from childhood to adulthood bridging the gap between psychiatry and neurology. His multilevel research interests combine developmental aspects with psychopathological behavior and neuropsychiatric symptoms of diseases with brain structure and brain function as well as biochemical and psychophysiological parameters and molecular aspects.

Manfred Gerlach was born on May 10, 1954, in Hammelburg, Germany. He studied chemistry and pharmacy at the University of Würzburg, Germany. He earned the doctorate in 1983. He worked as Scientific Assistant at the Clinical Neurochemistry, Dept. of Neurology, University Würzburg (1984-1987) and at the Dept. of Neurology, University Bochum (1987-1992). In 1991, he reached habilitation in clinical neurochemistry at the Dept. of Neurology (Head Prof. Dr. Horst Przuntek), University Bochum. In 1998, he became Associate Professor of Clinical Neurochemistry of the University Bochum. From 1992 to 2000 Manfred Gerlach joined as Scientific Assistant at the Clinical Neurochemistry Unit (chaired by Peter Riederer) at the Department of Psychiatry and

\footnotetext{
A. Warnke $\cdot$ M. Romanos ( $)$

Department of Child and Adolescent Psychiatry, Psychosomatics and Psychotherapy, University Hospital of Wuerzburg,

Würzburg, Germany

e-mail: Romanos@kjp.uni-wuerzburg.de

P. Riederer

Department of Psychiatry, Psychosomatics and Psychotherapy, University Hospital of Wuerzburg, Würzburg, Germany
}

Psychotherapy at the University of Würzburg. Since 2000, he is Head of the Clinical Neurobiology and Therapeutic Drug Monitoring Section of the Department of Child and Adolescent Psychiatry, Psychosomatics and Psychotherapy University of Würzburg with Andreas Warnke (until 2012) and Marcel Romanos (since 2012).

Manfred Gerlach initiated and performed a large number of research projects. His research was funded by the German research funding organisation (DFG) and the German Ministry of Education and Research (BMBF). Most projects were performed in close cooperation with his friend and scientific partner Peter Riederer. Colleagues of national and international scientific groups of departments of psychiatry and neurology and of child and adolescent psychiatry are partners in his many research projects. His methodological approach includes experimental methods in the field of biochemistry, pharmacology, genetics, proteomics and brain imaging.

His major research topics and findings include:

- Major scientific interests at the Clinical Neurochemistry Unit (Head: Peter Riederer) were based to elucidate the role of oxidative stress in neurodegenerative disorders as well as experimental and clinical trials in the field of neuroprotection and disease modification. Oxidative stress, iron toxicity and research on neuromelanin were and still are the main topics of interest. Based on this knowledge, the development of biomarkers for Parkinson's disease, attention deficit-/hyperactivity disorder (ADHD) and schizophrenia is of major interest. Manfred Gerlach contributed to these topics with ideas, methodological input and organizational concepts. More than 140 original publications were the result of this fruitful scientific cooperation in the field of neurodegeneration in general and Parkinson's 
disease in particular. Even after 25 years of intensive cooperation with Peter Riederer, this scientific coincidence is still active! And there are still fruitful collaborations with Kay Double, Katrin Marcus, Carsten Scheller, Daniela Berg and others to enroll the mysteries of neuromelanin.

- In ADHD, he put his focus of research on the association of the core symptoms with (a) molecular genetic conditions (with e.g., Tobias Renner, Susanne Walitza, Peter Lesch), (b) with abnormalities of brain function with and without stimulant medication (e.g., with Marcel Romanos, Susanne Walitza), (c) with specific features of the human ability to smell (with Romanos). Behind these very different research pathways, he followed one main scientific guideline: the exploration of the dopaminergic system with regard to its significance for the etiology and psychopharmacological treatment of ADHD symptoms.

- In autism spectrum disorder, he stimulated in search of biological markers studies on proteomics (with Regina Taurines, Johannes Thome).

- He reinforced studies on molecular genetic correlates of obsessive-compulsive disorder (with Susanne Walitza).

- He initiated international multicenter studies on therapeutic drug monitoring in child and adolescent psychiatry providing a means for improved pharmacovigilance and patient safety (with Mehler-Wex, Karin Egberts, Marcel Romanos).

- He now encourages and supports novel translational projects combining functional imaging and animal models in conjunction with human pathophysiology.

Manfred Gerlach is genius in the organization and performance of scientific meetings, national and international congresses many of them in partnership with Peter Riederer. Educational translation has been the basis of those scientific congresses, symposia and workshops. Manfred Gerlach has been and still is a great help in organizing such conferences: European Society of Clinical Neuropharmacology (ESCNP) 1995; 1. Congress of the German Parkinson's Disease Society (DGP) 1999; 16. International Congress of the Association of Parkinson's Disease and Related Disorders (IAPRD) 2005 to mention important ones. In 2007, he organized with Andreas Warnke and Peter Riederer the 1st World Congress on ADHD in Würzburg, and he was one of the main initiators of the World Federation of ADHD. Each of the World Congresses on ADHD in Berlin, Vienna and Milano brought together up to more than 2,000 participants from up to more than 80 countries-in each of these meetings Manfred Gerlach was highly successful as chairman of the scientific committee. He is engaged in different boards of scientific organizations and highly appreciated because of his extraordinary business competence and skill: e.g., he is member of the board of AGNP, DPG, GESENT e.V., World Federation ADHD and Association for the Performance of Scientific Conferences e.V.

As a teacher Manfred Gerlach presents excellent lectures to his students and on national and international meetings. He has the ability to make people understand even most complex scientific facts. Extraordinary is his engagement in the mentoring program of the Faculty of Medicine at the University of Würzburg. Up to now: of his former 9 mentees, 6 already reached habilitation, and 5 of them professorship and until now 4 former mentees became head of university clinical and/or research departments. Manfred Gerlach belongs to those mentors who support selflessly, reinforces and enjoys the success of mentees and colleagues. He is able to recognize the abilities and interests of a mentee and thus to adjust his stimulating ideas to the individual mentee. He is mentor not only with regard to research topics but also, if wanted by the mentee, to personal and family matters. His extraordinary competence as researcher in combination with his ability for trustful and supportive friendship is one main source of his own scientific success. It was his idea and initiative to establish the "August Homburger Price" of the University of Würzburg for excellent research in the field of biological child and adolescent psychiatry, and he initiated the "Heinrich Hofmann Medal" of the World Federation of ADHD for extraordinary and excellent clinical and scientific contributions for the understanding and treatment of ADHD. Manfred Gerlach is not only a partner in science but also a friend because of his musical engagement, his profound interest in literature, and his social engagement and sponsorship as member of the Lions Club Hammelburg, whose president he was for several times.

The volume of scientific publications includes more than 340 articles including handbook articles in renowned scientific journals in the fields of neurology, neuropharmacology and psychiatry. He is chief editor of the ADHD journal and member of the advisory board of Pharmacopsychiatry and "Zeitschrift für Kinder- und Jugendpsychiatrie und Psychotherapie". His book on Parkinson disease written in cooperation with Peter Riederer and Hans Reichmann in fourth edition is standard. The textbook on psychiatric drugs in childhood and adolescence (2nd edition), edited by Manfred Gerlach and colleagues, has now been translated into English. An important result of his research are three co-inventorships of patent applications. In 2006, he was honored with the Senator-Doktor-FranzBurda-Award.

His family support is crucial basis of all his activities. The support by his wife Rosi and by his family is 
inestimable. Rosi is a continuous source of reinforcement and support and of the extraordinary hospitality of the "Haus Gerlach".

This volume is filled with articles of friends, scientific colleagues and co-workers of Manfred Gerlach. The papers reflect the broad range of research topics, initiated and influenced by the work of Manfred Gerlach. The volume is dedicated to him on the occasion of his 60th birthday. We congratulate, and are thankful for his exceptional collegiality and friendship. 